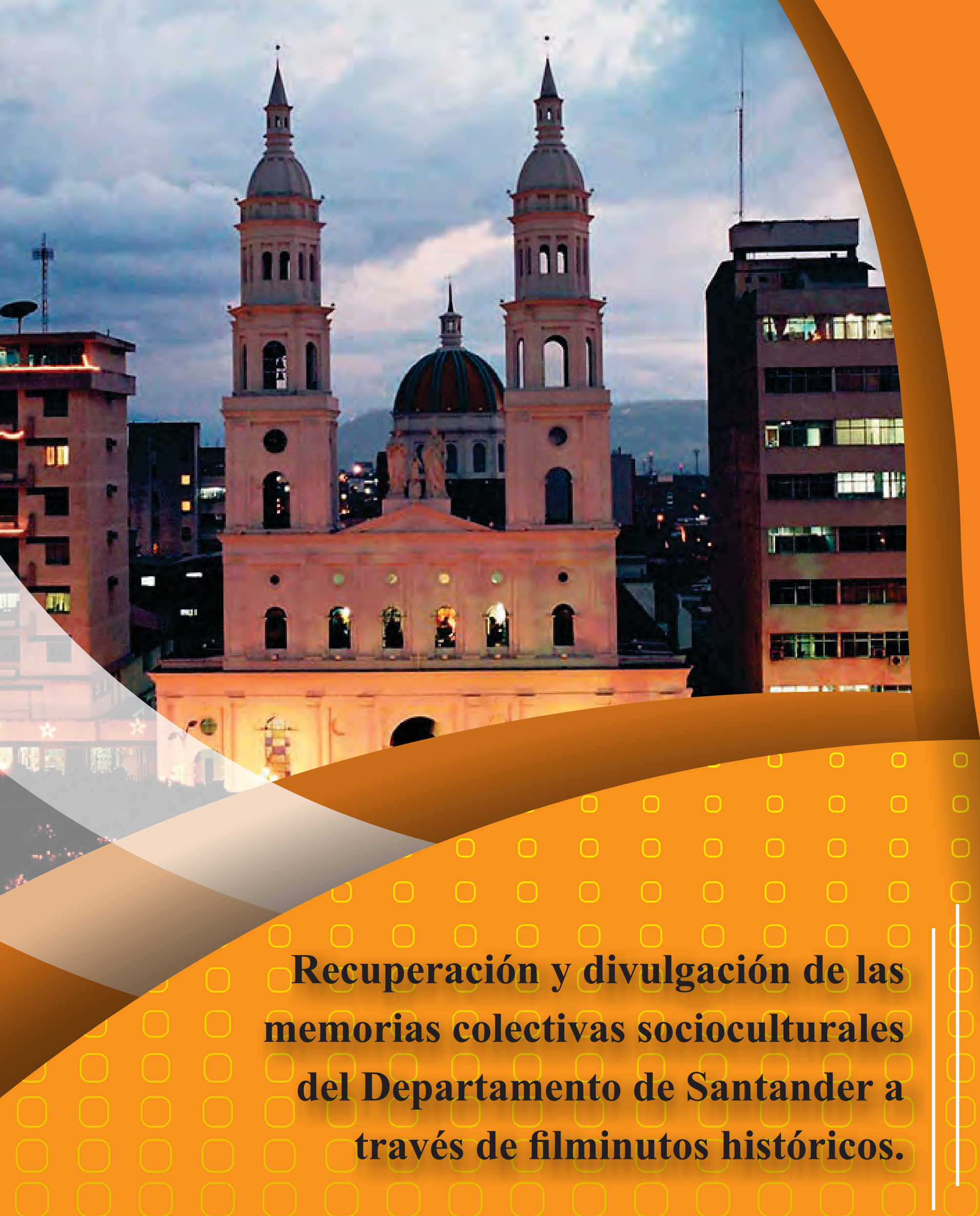




\title{
Recuperación y divulgación de las memorias colectivas socioculturales del Departamento de Santander a través de filminutos históricos. ${ }^{1}$ \\ Recovery and Dissemination of the Socio-Cultural Collective Memories of the Department of Santander on Historical Short Videos.
}

\author{
Ana Milena Valdés Peña ${ }^{2}$, María Olga Largacha Martínez ${ }^{3}$, Martha Lucía Herrera Leal ${ }^{4}$. \\ ${ }^{1}$ Universidad Manuela Beltrán-ITAE, Colombia.
}

Artículo recibido en el mes de Agosto de 2013; artículo aceptado en el mes de Noviembre de 2013.

Citación del artículo: Valdés, A., Largacha, M. \& Herrera, M. (2013). Recuperación y divulgación de las memorias colectivas socioculturales del Departamento de Santander a través de filminutos históricos. I+D Revista de Investigaciones, 2 (2), 20-28.

\section{Resumen}

Esta investigación tuvo como objetivo la generación de un dispositivo tecnológico informático virtual denominado filminuto o video de corta duración, como mecanismo de divulgación y recuperación del patrimonio cultural santandereano y como medio multimedial de fácil acceso para el público local y mundial en plataformas de internet, bajo el concepto de museo virtual. Este trabajo de investigación tecnológica aplicativa, parte del Grupo de investigación en Artes, Comunicación y Diseño (GIACODI) de la UMB-ITAE y se desarrolla dentro del Museo Virtual del Patrimonio e Identidad Cultural de Santander (MVPICS) de la Universidad Manuela Beltrán (UMB)-ITAE, y abarca la caracterización de

\footnotetext{
${ }^{1}$ Artículo de investigación tecnológica, de enfoque cualitativo, resultado de un proyecto de investigación culminado, desarrollado en el grupo de investigación en Artes, Comunicación y diseño GIACODI y fue financiado por la Universidad Manuela Beltrán-ITAE de la ciudad de Bucaramanga (Colombia). Dirección: Calle de los estudiantes 10-20, Ciudadela Real de Minas. PBX: 6525202.

${ }^{2}$ Profesional en Diseño de Modas, Fundación Universitaria del Área Andina. Especialista en Administración, Planeación y Desarrollo de la Investigación, Universidad Manuela Beltrán. Docente-investigador del grupo: Artes, Comunicación y diseño GIACODI. Universidad Manuela Beltrán-ITAE de la ciudad de Bucaramanga (Colombia). Dirección: Calle de los estudiantes 10-20, Ciudadela Real de Minas. PBX: 6525202. Correo electrónico: ana.valdes@itae.edu.co; annyvaldes_4@hotmail.com

${ }^{3}$ Maestro en Bellas Artes, Universidad Industrial de Santander. Especialista en Pedagogía del Diseño, Universidad Nacional de Colombia. Docente-investigador del grupo: Artes, Comunicación y diseño GIACODI. Universidad Manuela Beltrán-ITAE de la ciudad de Bucaramanga (Colombia): Dirección: Calle de los estudiantes 10-20, Ciudadela Real de Minas. PBX: 6525202.

${ }^{4}$ Comunicadora Social - Periodista, Universidad Autónoma de Bucaramanga. Estudios internacionales en desarrollo y exclusión social, Instituto Universitario de Estudios del Desarrollo, Ginebra-Suiza. Docente-investigador del grupo: Artes, Comunicación y diseño GIACODI. Universidad Manuela Beltrán-ITAE de la ciudad de Bucaramanga (Colombia). Dirección: Calle de los estudiantes 10-20, Ciudadela Real de Minas. PBX: 6525202.
} 
costumbres, vestimentas, objetos de uso, ambientes, expresiones lingüísticas, utilizadas en el departamento de Santander en los períodos históricos de: la conquista, la colonia, la república, el estado federal y el Gran Santander. Los resultados muestran once piezas fílmicas históricas dentro de los períodos citados en el departamento de Santander.

Palabras clave: filminutos históricos, historia, Departamento de Santander, patrimonio cultural.

\section{Abstract}

The purpose of this study was the making of a virtual technological device: a historical short videos, in order to disseminate the cultural heritage of the Department of Santander (Colombia) as an internet - easy - to - access multimedia device, for the people in the region and around the world, under the concept of virtual museum. This technological - applied research was formulated at the investigation group: Artes, Comunicación y Diseño (GIACODI) of Manuela Beltrán (UMB)-ITAE University, at the cultural heritage and identity of the Department of Santander Virtual Museum (MVPICS), whose objectives includes the characterization of customs, outfitting, used objects, environments and idiomatic expressions utilized at the Department of Santander through its historical periods at the Conquer, the Colony, the Republic, the Federal State and the Great Santander. The results are eleven historical short videos about aforementioned aspects into those periods in this region of Colombia.
Keywords: historical short videos, Department of Santander, cultural heritage.

\section{Introducción}

Los objetos utilitarios y el vestuario utilizados por el hombre, recrean su contexto histórico, político, económico y socio cultural, dando cuenta de sus formas de vida y de su expresión individual y colectiva en la civilización.

Los museos han albergado mundial-mente muestras de dichos elementos, que son exhibidos a la población, con el propósito de mantener vigentes los usos, modos y modas del hombre en diferentes momentos históricos, por lo cual son agentes de preservación de la memoria histórica de los pueblos. En el caso del departamento de Santander, en los 43 museos existentes, se exhiben de manera muy precaria algunas piezas de indumentaria y objetos utilitarios propios de los diferentes momentos históricos de la región (excluyendo las colecciones patrimoniales santandereanas, un elemento importante de la memoria y la identidad cultural del territorio), con el consecuente resultado en términos de difusión, que además, se halla circunscrito a lo tangible y a la experiencia directa de manera exclusiva para el usuario.

El auge de las nuevas tecnologías de la información y la comunicación, ha otorgado importancia a nuevas formas de acceso a la misma, a través de medios virtuales y multimediales que se han introducido en la museística, privilegiando la experiencia 
intangible e indirecta, mediatizada por dichos medios de imagen, sonido, movimiento, que permiten exhibir piezas originales al público de manera continua, sin restricciones de tiempo ni espacio y de forma remota, abriéndose así un espacio para la divulgación de las tradiciones, costumbres, artefactos y demás, que cuentan con valor histórico y cultural.

En el caso de la memoria colectiva indumental, objetual y social, relacionada con el patrimonio e identidad santandereanos, los registros audiovisuales juegan un papel importante para difundir sus transformaciones, movimientos, adaptaciones y evolución histórica, democratizando su acceso. Estas alternativas, sin embargo, han sido muy poco promovidas en el país y en la región, siendo así uno de los campos de expansión y desarrollo de la actividad museística y cultural de mayor dinamismo en el contexto de la cibersociedad. En este sentido, el Museo Virtual del Patrimonio e Identidad Cultural de Santander (MVPICS) de la Universidad Manuela Beltrán (UMB)-ITAE, ha sido una iniciativa que, basada en la investigación, ha implementado la difusión museística virtual, iniciando por la vestimenta, en un período de la historia colombiana desde 1810 hasta 2010, es decir, desde la declaración de independencia, hasta el actual período de la República de Colombia, para lo cual se ha apoyado en el trabajo del Grupo de Investigación en Artes, Comunicación y Diseño (GIACODI) de la UMB-ITAE.

Con este mismo propósito de la difusión museística virtual de la identidad cultural santandereana, se ha propuesto dar a conocer los diferentes aspectos relacionados con la misma, de lo cual se ha derivado la siguiente pregunta de investigación: ¿Cómo hacer posible el acceso virtual, libre e interactivo del público a las manifestaciones socio culturales santandereanas, asociadas con la vestimenta y objetos de uso, utilizados en la Conquista, la Colonia, la Independencia, la República, el Estado Federal y el Gran Santander?

\section{Método}

\section{Tipo de estudio.}

Se siguió un tipo de investigación tecnológica aplicativa, sobre el filminuto como medio para la difusión de los aspectos históricos, socio culturales, en diferentes períodos históricos del departamento de Santander.

\section{Participantes}

Docentes, estudiantes y personal de apoyo del Grupo de Investigación en Artes, Comunicación y Diseño (GIACODI) de la UMB-ITAE que participan en la producción de los filminutos. 
Tabla1. Distribución de los participantes en el proyecto según su rol y film a realizar

\begin{tabular}{|c|c|c|}
\hline \# Film & Nombres de los autores & Roles \\
\hline \multirow[t]{6}{*}{1} & Santiago Vallejo & Dirección \\
\hline & & Cámara y Edición \\
\hline & Omar Vega & Producción \\
\hline & Jair Coronado & Asist. Producción \\
\hline & Eric Ortega & Sonido \\
\hline & Laura Johana Arias Lozano & $\begin{array}{c}\text { Dirección de Arte } \\
\text { Vestuario }\end{array}$ \\
\hline \multirow{7}{*}{2} & Iván León & Producción y Dirección \\
\hline & Sergio Reyes & \\
\hline & Juan Navas & \\
\hline & Jimena Bueno & Dirección de Arte \\
\hline & Dianny Roxana Romano & Vestuario \\
\hline & Mónica Galvis & Asist. Dirección de Arte \\
\hline & Lina Saavedra Sanabria & \\
\hline \multirow[t]{8}{*}{3} & Oscar Peñuela & Dirección \\
\hline & Julián Correa & Dirección \\
\hline & & Cámara y Edición \\
\hline & Dulce María Cartagena & Sonido \\
\hline & María Fernanda Pinto & Dirección de Arte \\
\hline & Wilmer Cadena & \\
\hline & Jimena Bueno & Vestuario \\
\hline & Dianny Romano & \\
\hline \multirow[t]{4}{*}{4} & Adriana Suárez & Producción Edición \\
\hline & & Música \\
\hline & Julián Correa & Cámara \\
\hline & Jhon Hernández & Asist. Producción \\
\hline \multirow[t]{6}{*}{5} & Jackson J. Gómez & Dirección y Fotografía \\
\hline & Juan Carlos García & Producción \\
\hline & Mario A. Galeano & Edición \\
\hline & Jackson J. Gómez & \\
\hline & Laura Johana Arias Lozano & Dirección de Arte \\
\hline & & Vestuario \\
\hline \multirow[t]{16}{*}{6} & Oscar Olney Fernández & Dirección \\
\hline & Katherine Orcasitas & Producción \\
\hline & & Asistente Dirección \\
\hline & Nikolai González & Fotografía y Cámara \\
\hline & Oscar Olney Fernández & \\
\hline & Jonathan Jaimes Ferreira & Sonido \\
\hline & Vanessa Mae - The V iolin Fantasy & Música \\
\hline & Katherine Orcasitas & Edición \\
\hline & Nikolai González & \\
\hline & Oscar Olney Fernández & \\
\hline & Andrea Morales & Dirección de Arte \\
\hline & Constanza Cadena & \\
\hline & Natalia Guevara & \\
\hline & Mónica Esthela P. Rodríguez & Vestuario \\
\hline & Jessica M. Ardila & \\
\hline & Mayra Alejandra A. Delgado & \\
\hline \multirow[t]{7}{*}{7} & Vanessa Amaya & Dirección \\
\hline & Tatiana Valencia & Producción \\
\hline & Jackson J. Gómez & Dir. Fotografía \\
\hline & Mario Forero & Dir. Actores \\
\hline & Mario Galeano & Sonido \\
\hline & Laura Johana Arias Lozano & Dirección de Arte \\
\hline & & Vestuario \\
\hline \multirow[t]{6}{*}{8} & Sandra Fuentes & Dirección \\
\hline & Álvaro Angarita & Cámara y Montaje \\
\hline & Laura Churio & Producción \\
\hline & Henry Moreno & Asistente de Producción \\
\hline & Andrea Villamizar & Dirección de Arte \\
\hline & Geraldine Ramírez & \\
\hline \multirow[t]{2}{*}{9} & Any Paola León & Dirección \\
\hline & Carolina Garavito & Producción \\
\hline
\end{tabular}

Fuente: Autor 


\section{Materiales e instrumentos}

\section{programa de diseño de ambientes virtuales.}

Por medio de este programa informático se recrean las escenas insertas en una línea de tiempo dentro del (MVPICS); sobre una línea de tiempo emerge una serie de íconos o botones que, al activarse, permiten al usuario acceder a las salas correspondientes a cada época referida o salas temáticas. La línea tendrá de manera paralela, la barra menú o línea del tiempo, con la información referencial sobre eventos históricos a los que el usuario podrá acceder, dependiendo del itinerario o recorrido que decida tomar. $\mathrm{La}$ temporalidad de la misma es correspondiente a la historia fragmentada por los períodos de conquista, colonia, independencia, república, estado federal y Gran Santander.

\section{utilería, vestuario, logística, ambientación}

Logística y coordinación en aspectos relativos a diseño y desarrollo de propuestas de vestuario y styling, en cuanto al producto audiovisual dinámico, para la presentación de la indumentaria histórica por medio de los elementos de carácter utilitario, en la realización de los filminutos.

\section{documentación para texto y guión en los filminutos.}

Consulta de fuentes primarias y secundarias, exploración de la bibliografía pertinente a cada objeto museal, cartografía, inventarios existentes, investigaciones previas, fotografías antiguas. Logística y coordinación en aspectos relativos a la ambientación, por medio de los elementos de carácter utilitario en la realización de los filminutos y la gestión desde el semillero
SEDIF de los apoyos, con el elemento de carácter utilitario en la ambientación de los espacios.

\section{producción de los filminutos.}

PRTV lleva a cabo la totalidad de las fases que llevan a la materialización de los filminutos mediante la preproducción, producción y postproducción del material audiovisual histórico.

\section{Procedimiento}

Se definieron los contenidos, forma de navegación y utilización del ambiente virtual del museo para el usuario potencial. Para ello se estableció, que primero accedía a las salas a través de un ícono de un traje alusivo a una época o período de la historia del departamento de Santander. Una vez el usuario accede a las salas, por medio de la imagen de un traje correspondiente a la época o momento en la línea del tiempo del menú de navegación general, tendrá la sensación de estar recorriendo la sala de un museo, encontrando los diferentes elementos que usualmente se encuentran en un museo real.

Cada una, presenta al usuario la posibilidad de interactuar con los diferentes momentos y contextos relativos al traje, al momento histórico, detalles del uso y del contexto del traje presentado mediante un corto audiovisual (ver tabla 2), permitiendo al usuario acceder a la información pertinente de la sala, en diferentes itinerarios organizados a manera de visita museal. El usuario ingresa entonces a la sala, teniendo a disposición vistas generales de los trajes y objetos de la misma. Al acercarse en su recorrido a cada traje u objeto, éste se constituye 
en el hipervínculo que permite acceder a la visión $3 \mathrm{D}$ con rotación en 360 grados. Cada traje u objeto igualmente presenta, al ser recorrido con el mouse, una serie de hipervínculos que remiten al usuario a información sobre cada pieza a manera de anécdota, o bien, información detallada de la pieza en sí. El usuario estará en libertad de elegir la pieza u objeto que desea observar; de la misma manera podrá acceder o salir de la sala cuando lo desee. Cada sala recrea el contexto físico de la época de los trajes, o sea, si los trajes son de la conquista, el espacio deberá presentar referentes concretos a la arquitectura y espacios de la época, con los materiales, colores, formas, y detalles típicos de ella.

Tabla 2. Épocas históricas y denominación en cada film.

\begin{tabular}{ccc}
\hline Film & Denominación & Época \\
\hline 1 & "La Batalla Perdida" & 1819 \\
2 & "Tradición oral: Brujas de Girón” & 1825 \\
3 & "Error Fatal" & 1850 \\
4 & "Nacimiento de la Cerveza en Bucaramanga" & 1869 \\
5 & "Creación del Estado Soberano de Santander" & 1861 \\
6 & "La Culebra Pico de Oro" & 1870 \\
7 & "Inicios de la Revolución de los Comuneros" & 1871 \\
8 & "Industria Tabacalera de Santander" & 1876 \\
9 & "La Mujer Motor" & 1882 \\
10 & "Club del Comercio" & 1905 \\
11 & "La Guerra de los Mil Días" & 1903 \\
\hline
\end{tabular}

Fuente: Autor

Se definieron las funciones y productos, junto con sus cronogramas y responsables de los trajes, utilería, escenografías, locaciones de filmación y aspectos logísticos involucrados en el tema.

Se llevaron a cabo las búsquedas documen- tales y se emprendieron las investigaciones sociológicas, políticas, etnográficas, fotográficas y demás, para contextualizar los contenidos de los filminutos.

Se llevó a cabo la asignación de épocas históricas para los anteriores estudios documentales, a los equipos encargados.

Se procedió a organizar los guiones y libretos a desarrollarse en los filminutos y todo el trabajo de preproducción, producción y postproducción de televisión de los filminutos.

Se analizaron diferentes aspectos asociados con las expectativas de producción del material audiovisual histórico.

\section{Resultados}

Se obtuvieron once piezas audiovisuales, puestas en valor mediante el uso innovador de las tecnologías de la información y la comunicación, que se exponen como propuestas inmateriales que recuperan el patrimonio y recrean los diversos escenarios que conforman sucesos representativos de la región, durante el bicentenario de su independencia, hacia lo que sería en el entorno virtual; los temarios giran en torno a hechos tales como: la batalla de Charalá en el puente de Pienta que impidió el refuerzo militar de los conquistadores (1819), Los caminos de Geo Von Lenguerke por Santander (1850), José Lámbole, la creación de una cervecería en el municipio de Floridablanca (1869), José Bonifacio Aquileo Elías Parra Gómez y la creación del Estado Soberano de Santander (1861), comerciantes y artesanos en 
Bucaramanga "Los Pico de Oro"(1870), inicios de la revolución comunera en el Socorro (1871), el posicionamiento que tuvo el tabaco fabricado en el municipio de San Juan Girón (1876), el primer discurso de una bachiller en Bucaramanga (1882), el Liceo de Soto, sus inicios hasta 1905 (pre 1872), La Guerra de los Mil Días en la República de Colombia (1903), la existencia de Sacerdotisas o brujas en el municipio de Girón, Santander (1925).

Cada una de las piezas recrea vestimentas, objetos, ambientes, circunstancias socio culturales, costumbres y tradiciones, así como modos lingüísticos particulares de cada contexto socio histórico, a través del cual, de manera rápida pero sustanciosa, el usuario puede hacerse una idea acerca de cómo vivían las personas en cada una de las épocas descritas en el departamento de Santander.

\section{Discusión}

Tomando como punto de partida que la imagen se convierte en un propicio elemento que reconstruye contextos sociales, geográficos, psicológicos y humanos, las piezas fílmicas históricas producidas introducen en el museo virtual, registros audiovisuales que recrean hechos históricos de la vida de los habitantes de la región santandereana, que van más allá de la socialización de hechos cronológicos que hacen parte de la memoria colectiva y dejan al conocimiento del usuario elementos del contexto, entre los que se encuentra el lenguaje verbal y corporal, objetos, sentimientos. Es pertinente retomar el aporte conceptual de Santos Zunzunegui, en el que analiza la importancia de la imagen como recurso, que va más allá de un montaje actoral. "Hoy en día también sucede algo similar, en la medida en que nuevas formas de arte se "rozan" con las que hasta ahora hemos venido considerando especificamente filmicas. Se habla sin parar en ciertos círculos del post cine y de la museificación del cine, ya que, cada vez más, el cine se exhibe en los museos y es utilizado como mecanismo artístico en combinación con otras prácticas no cinematográficas".

En el museo virtual se consideró pertinente la utilización del audiovisual como enlace entre el ícono de la época histórica y el detalle de la exposición del traje correspondiente, sus características en cuanto al diseño, la composición, tipo de materiales, usos y ocasiones de las mismas, agrupadas cronológicamente en las épocas comprendidas entre $1.800 \mathrm{al}$ 2.010, garantizando la preservación y sostenimiento de la riqueza cultural regional, la gestión del conocimiento cultural y patrimonial del departamento en constante evolución y cambio en torno a las apariencias estructurales, arquitectónicas, paisajísticas, objetuales y vestimentales, que por un lado se mantienen dentro del marco de la materia virtual como memoria y tradición, y por otro lado como proceso de promoción continua del conocimiento cultural.

Para quienes actúan en calidad de usuarios y generadores a su vez, de importantes vínculos con la identidad social y cultural santandereana, como principales protagonistas y multiplicadores a través del eco virtual, encontrarán en los filminutos la remembranza sobre la colectividad con grados de pertenencia, y en la labor de 
conservación de la heredad social, propenderán en el ejercicio continuo de apropiación, implicación, e interacción con parte del patrimonio cultural nacional, a través de la dinámica virtual .

\section{Referencias}

Balerdi, I. (2002). ¿Qué fue de la museología?. El caso de Quebec. Antigrama, 17.

Bellido, M. L. (2001): Arte, museos y nuevas tecnologías. Gijón: Editorial Trea.

Carrera, C. (2009). Evaluación TIC en el patrimonio cultural, Acción Cultura, Museos y Patrimonio, Barrcelona: Editorial UOC.

Century, M. (1995). Beyond the virtual museum: towards ART (augmented reality telepresence). Recuperado de:

http://www.music.mcgill.ca/ mcentury/Papers/ ART.html.

Falk, J., \& Dierking, L. (1992). The museum experience. Washington D.C.: Whalesback Books.

Fisher, P. (1991): Making and Effacing Art: Modern American Art in a Culture of Museums, Cambridge: Harvard University Press.

Gómez, A. (2000). El concepto de Heurística en las ciencias y las humanidades, ciudad de México: Siglo XXI Editores.
González, B. (2003): Museos de ciencia en las universidades: algunas reflexiones y una descripción, Revistade Museología, 27, 67-68.

Hackman, W. (1992): Wonders in one close shut: the educational potential of history of science museums. En Durant, J. (ed.), Museums and the public understanding of science. Londres: Science Museum.

Hein, G. E. (1998): Learning in the museum. Londres: Routledge.

Hernández, F. (2006). Planteamientos teóricos de la nueva museología. Gijón: Editorial Trea.

Hoptman, G. H. (1992): The virtual museum and related epistemological concerns. En Barret, E. (ed.): Multimedia, Hypermedia and the Social Construction of Knowledge. Cambridge: MIT. Press.

Karp,I. (ed.), Museums and Communications The Politics of Public Culture. Washington, D.C.: Smithsonian Institution Press.

Lord, B., Lord, G. D. \& Fullola, J. M. (1998). Manual de gestión de museos. Barcelona: Editorial Ariel.

McDonald, G. (1992): "Change and challenge: museums in the information society". Washington D.C.: Smithsonian Institution Press. 